\title{
Skeletal Muscles Modeling for Isogeometric Analysis
}

\author{
Linlin Li $i^{1 a}$, John Boss ${ }^{2 b}$ \\ ${ }^{1}$ Suzhou Industrial Park Institute of Vocational Technology, Suzhou, Jiangsu, China \\ ${ }^{2}$ Department of Mechanical and Industrial Engineering, the University of lowa, lowa City, IA, USA \\ aEmail: 75054637@qq.com bEmail: vickylyg@eyou.com
}

Keywords: Skeletal Muscle. Muscle Modeling. Isogeometric Analysis

Abstract. This research uses only surface control points to parameterize the 3D geometry of a muscle body. Extension from surface to interior is achieved using a harmonic interpolation in cross section and in the lateral direction using NURBS. This representation preserves geometric smoothness, and allow muscle fiber distributions to be easily parameterized. Utilizing this geometric method also provides the framework for implementing material properties using an interpolative-style scheme. Upon supplying constitutive models these models can be directly used in analysis.

\section{Introduction}

Isogeometric analysis, a generalization of finite element method, has the potential to satisfy the motivations of the computer graphics and biomechanical analysis of muscle modeling [1]. It integrates Computer Aided Design (CAD) and mechanical analysis by using the same geometric basis for both ${ }^{[2]}$. The most relevant modeling techniques is NURBS ${ }^{[3]}$. Skeletal muscles are topologically regular ${ }^{[4]}$, allowing each of them to be parameterized continuous using a set of parametric coordinates. It allows for easy parameterization of muscle fiber orientation and material property distribution along slender muscle bodies.

\section{Tensor-product Rod Method}

A method for parameterizing a unit cylinder starts with a rational Bezier circle, then extends the boundary representation into the interior of the circular domain. After utilizing Bezier circles for the cross-sectional representation, a NURBS centerline is used to bring the longitudinal component into the $3 \mathrm{D}$ rod representation, formally known as the tensor-product $\operatorname{rod}^{[1]}$. Combining in this manner gives the tensor-product rod

$$
x=\sum_{i=1}^{n} \sum_{j=1}^{m} T_{i}\left(\xi^{1} \xi^{2}\right) R_{j}(u) P_{i j}
$$

with $T_{i}\left(\xi^{1}, \xi^{2}\right)$ as the cross sectional representation and $R_{j}(\boldsymbol{u})$ as the axial representation. The domain in this case is defined by

$$
\Omega=D \otimes\left[u_{1}, u_{m+p+1}\right]=\left\{\xi^{1} \otimes \xi^{2} \otimes u:\left(\xi^{1}\right)^{2}+\left(\xi^{1}\right)^{2} \leq \mathbf{1}, u_{1} \leq u \leq u_{m+p+1}\right\}
$$

which is that of a cylindrical element with a NURBS knot span of $\left[u_{1}, u_{m+p+1}\right]$. 
In the present case each segment of the centerline can be thought of as an individual element. This divides the cylinder into sub-cylinders where each sub-cylinder corresponds to a non-empty knot span of $\left[u_{1}, u_{\mathrm{m}+\mathrm{p}+1}\right]$. With $n$ control points per reduced Bezier cross section and a centerline of degree $p$, each element in this case contains $n(p+1)$ control points.

\section{Isogeometric models of muscles}

\section{Surface geometry}

Each muscle's cross sections are compiled together and lofted into NURBS surface models. The cross sections are each rebuilt with 8 control points and the skeletal muscle shapes are terminated near their tendon/bone origination/termination locations. At these endpoint locations, 8 point objects are positioned in a single location. Each model contains a varied number of cross sections and number of total control points which depends on the overall length of each individual muscle. The lofting operations are done by the NURBS-based 3D CAD software, Rhinoceros.

\section{Fiber structure}

For finite element muscle models, fiber orientation will need to be generated and stored at each Gauss points ${ }^{[5]}$. In this way the parameterization is universal and can be applied to any muscle shape. Various fiber architectures can then be easily defined in the master rod and then mapped to the physical rod. The following are a few example formulations that can be applied to a model to accurately represent the various fiber orientations.

Parallel Fibers In the master rod, parallel fibers are straight lines parallel to the longitudinal axis ${ }^{[6]}$. The two coordinate directions associated with the cross-sectional representation of the tensor-product rod method $\left(\xi^{1}, \xi^{2}\right)$ are fixed. Utilizing the parametric nature of the centerline represented by a NURBS curve, the variable $u$ can be traced out in the longitudinal direction. Thus, in the physical domain, the fiber curve is represented by

$$
x(u)=T\left(\xi^{1}, \xi^{2}\right) R(u) P \quad u_{1} \leq u \leq u_{m+n+1}
$$

The tangent of this curve at any point defines the fiber direction at that point. In particular, fiber directions for points of interest within the tensor-product rod. An example of a parallel fibered muscle is the extensor carpi ulnaris. The muscle model is shown in Fig. 1(a), it is plotted utilizing the parallel fiber parameterization.

Helical Fibers The type that the twisting of parallel-fibered muscles along their longitudinal axis called helical ${ }^{[6]}$ in nature can be represented by

$$
x(u)=T(r \cos (\omega u+\emptyset), r \sin (\alpha u+\emptyset)) R(u), u_{1} \leq u \leq u_{m+n+1}
$$

with $r=\sqrt{\left(\xi^{1}\right)^{2}+\left(\xi^{2}\right)^{2}}$ and $\emptyset=\tan ^{-1}\left(\xi^{2} / \xi^{-1}\right)$. Here $\alpha$ is a constant that can modify the severity of the helical twist and twist direction. The helical direction is reversed by the use of a negative constant. Due to the longitudinal division into knot spans of the tensor-product rod, this type of representation can be implemented for an entire skeletal muscle or in conjunction with another type of fiber parameterization such as the parallel-fibered case. An example of a muscle with helical fibers is the brachioradialis, it is shown in Fig. 1(b) with a parallel fiber parameterization for the majority of muscle length and a helical-fiber parameterization applied where the muscle wraps around the brachialis muscle just above the elbow.

Convergent Fibers Convergent muscles do not necessarily terminate at a common tendon location as in the parallel case, but rather they may have multiple attachment points ${ }^{[6]}$. In the 
muscle model created, the tensor-product solid has a single origination and termination point. This means if fiber direction were assigned along the isocurves as described in the parallel fiber case, fiber direction would converge rapidly on the origination or termination point regardless of geometric shape. To remedy this, parameterization is used in which the fiber direction can converge or diverge from the isocurves, leading to fiber termination points at the walls of the tensor-product solid. The formulation for this procedure is depicted by the following representation

$$
x(u)=T\left(\xi^{1}(1+a u) \xi^{2}(1+a u) R(u) P \quad u_{1} \leq u \leq u_{m+n+1}\right.
$$

Here the variable $a$ is the convergence/divergence intensity of the particular skeletal muscle. A negative value for $a$ will cause the fibers to converge to the NURBS centerline of the tensor-product rod shape. A boundary condition is also used that disallowed fibers from passing through the skeletal muscles wall. An example of a muscle that is convergent in nature is the pronator teres. The fiber parameterization model of this muscle which drives pronation of the forearm can be seen in Fig. 1(c). Utilizing the above formulation for convergent muscles. It should be noted that as the fiber parameterization approaches the boundary the solution becomes unstable so care should be taken to avoid this phenomena.

Pennate Fibers Pennate-fibered muscles have one or more tendons running the through the bulk of the muscle body and the fibers are attached to the tendons at a distinct angle relative to the line-of-action of the muscle ${ }^{[6]}$. A pennate fibered muscle represented in the tensor-product rod domain can be defined by

$$
x(u)=T\left(b\left(u-u_{0}\right)+c_{1} \xi^{2}\right) R(u) P_{2} \quad u_{1} \leq u_{i} \leq u_{m+n+1}
$$

with $c=\xi^{1}-b z$.The degree of pennation is determined by the slope factor $b$ and $z$ is the longitudinal location of the point which fibers are running through in each element. Switching signs of the slope factor will switch pennate direction. Of course pennation can also be implemented in the $\xi^{2}$ direction, as well as any combination of the two. An example of a pennate fibered muscle is

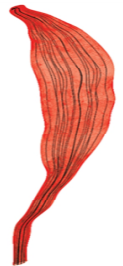

(a)

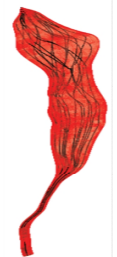

(b)

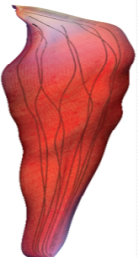

(c)

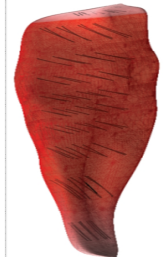

(d)

Fig. 1: Example of fiber architecture. (a)parallel fibers; (b) helical fibers; (c) convergent fibers; (d) pennate fibers

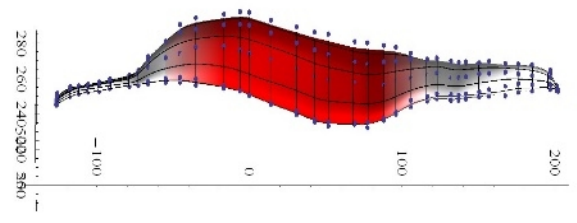

Fig.2: Visually assigned tendon/muscle fiber properties and their cross-sectional breakdown

the anconeus. The anconeus muscle model and pennate fiber parameterization is shown in Fig. 1(d).

Computing Fiber Direction A distinct feature of this parameterization is that the fiber lines are obtained by restriction. Since fibers are parameterized by curve

$$
\xi^{1}=\xi^{1}(s), \xi^{2}=\xi^{2}(s), u \xi^{3}=u=\xi^{3}(s)
$$

that is at a material point designated by " $s$ ", $\mathrm{x}=\mathrm{x}(s)$, the tangent of the curve is $\frac{d \mathrm{c}(s)}{d s}$. Note that no additional control points were introduced. 


\section{Heterogeneous properties}

The muscle-tendon connection is a gradual change of properties ${ }^{[7]}$. Within the tensor-product framework, the variation of properties can be implemented using the tensor product presentation. If $C$ is a particular material constant, the field values of which can be represented by

$$
a(u)=C(u)=\sum_{i=0}^{n} N_{i, p}(u) a_{i}
$$

Here $a_{i}$ are material components that can be arbitrarily determined to match any muscle's specific muscle-tendon. The parameters can be thought as varying only in the longitudinal direction. This situation can be obtained simply by assigning the same values to the control parameters in a cross section.

Fig. 2 shows a visual representation of a varying property the muscle-tendon complex. The boundaries for NURBS elements are $C_{p-1}$ continuous, meaning control points within the NURBS framework are shared among neighboring elements, exhibiting the globally smooth nature of the tensor-product rod and heterogeneous property distribution that is desired.

\section{Conclusion}

Within the framework of the tensor-product rod method, it has been shown that an anatomically accurate, analysis-ready muscle model can be produced. This muscle model satisfies the three main criteria that fully characterize an analysis-ready model: geometric description, fiber parameterization, and heterogeneous property assignment.

\section{Acknowledgment}

This work is supported by High Level Key Major Construction Project of Jiangsu Province (2017).

\section{References}

[1] Jia Lu and Xianlian Zhou. Cylindrical element: Isogeometric model of continuum rod. Computational Methods in Applied Mechanics and Engineering, 2000.

[2] Scott P. Delp and J. Peter Loan. A computational framework for simulation and analysis of human and animal movement. Computing in Science and Engineering, 2:46-55, 2000.

[3] Thomas J.R. J. Austin, Hughes and Yuri Bazilevs. Isogemetric analysis: Toward integration of cad and fea. Chichester: John Wiley and Sons, Ltd., 2009.

[4] Timmons Michael J Martini, Frederic H and Robert B Tallitsch. Human anatomy. s.l.: Benjamin Cummings, 123-251, 2008.

[5] Carole Blanc and Christophe Schlick. Accurate parametrization of conics by nurbs. Computer Graphics and Applications, 1996.

[6] Jia Lu. Circular element: Isogeometric elements of a smooth boundary. Computer Methods in Mechanics and Engineering, 2009.

[7] Oomens, C.W.J. Finite element modelling of contracting skeletal muscle. Philosohpical Transactions of the Royal Society of London, pages 1453-60, 2003.

[8] John C. Gardiner and Jeffrey Weiss. Simple shear testing of parallel-fibered soft tissues. Journal of Biomechanics, 123:170-175, 2001. 